\title{
Impact of a Pharmacist-Led Antimicrobial Stewardship Program on the Number of Days of Antimicrobial Therapy for Uncomplicated Gram- Negative Bacteremia in a Community Hospital
}

\author{
Tetsuya Fukuda ${ }^{1,2}$, Kentaro Tanuma ${ }^{2}$, Satoru Iio ${ }^{2}$, Jumpei Saito ${ }^{1}$, Makoto Komura ${ }^{1}$, Akimasa Yamatani
} 1

1. Department of Pharmacy, National Center for Child Health and Development, Setagaya-ku, JPN 2. Department of Pharmacy, National Hospital Organization Nishisaitama-Chuo National Hospital, Tokorozawa-shi, JPN

Corresponding author: Tetsuya Fukuda, tetsuyama@live.jp

\section{Abstract \\ Introduction}

The need for pharmacist-led antimicrobial stewardship programs (ASP) is increasing.

\section{Objective}

We performed a retrospective study to assess whether pharmacist-led ASPs can decrease the duration of treatment for uncomplicated gram-negative bacteremia among patients admitted in a community hospital.

\section{Methods}

This research was conducted at a 325-bed regional general hospital in Japan, from January 2013 to June 2015. There are no infectious diseases specialists affiliated with the hospital. The outcomes of the pharmacist-led ASP group, who received pharmacist intervention, and the control group, who did not receive pharmacist intervention, were compared. The study included patients aged 18 years or older who were diagnosed with gram-negative bacteremia. The pharmacist performed an antimicrobial time-out at 72 hours after blood culture collection and optimized treatment based on the patient's clinical response and test results. The primary outcome was the duration of antibiotic treatment.

\section{Results}

In total, 34 patients in the pharmacist-led ASP group and 32 in the control group were included in the final analysis. The median number of days of antimicrobial treatment was 8 (interquartile range [IQR]: 7-14) days in the pharmacist-led ASP group and 14 (IQR: 10-15) days in the control group. The number of days of antimicrobial treatment significantly reduced in the pharmacist-led ASP group $(\mathrm{p}<0.001)$. The deescalation rates were 11 (32.4\%) cases in the pharmacist-led ASP group and 4 (12.5\%) cases in the control group. Hence, the trend was higher in the pharmacist-led ASP group than in the control group $(p=0.08)$.

Review began 03/26/2021 Review ended 04/22/2021 Published 04/22/2021

๑) Copyright 2021 Fukuda et al. This is an open access article distributed under the terms of the Creative Commons Attribution License CC-BY 4.0., which permits unrestricted use, distribution, and reproduction in any medium, provided the original author and source are credited.

\section{Conclusion}

The pharmacist-led ASP reduced the number of days of antimicrobial therapy for uncomplicated gramnegative bacteremia among patients admitted in a community hospital without an infectious diseases specialist.

Categories: Medical Education, Infectious Disease, Other

Keywords: adolescent, bacteremia, pharmacists, physicians, specialization, antimicrobial stewardship, community hospital, team medicine

\section{Introduction}

The proportion of drug-resistant bacteria is increasing worldwide. This phenomenon is attributed to the inappropriate use of antimicrobial agents. Moreover, the development of new antimicrobial agents has been decreasing. Thus, drug-resistant bacteria have become an issue, and antimicrobial stewardship programs (ASP) [1] have been attracting attention.

A previous study has shown that pharmacists can lead ASPs [2]. In Japan, there are only a few infectious diseases specialists [3]. Therefore, the need for pharmacist-led ASP, which can improve the quality of medical care for Staphylococcus aureus bacteremia [4-5], is increasing. Moreover, a previous research has presented the health and economic benefits of pharmacist-led ASP among patients admitted in a community hospital in Japan [6]. However, only a few reports have shown that pharmacist-led ASPs reduced 
the number of antimicrobial days.

Hence, we aimed to perform a retrospective study to assess whether pharmacist-led ASPs can decrease the duration of treatment for uncomplicated gram-negative bacteremia among patients admitted in a community hospital without an infectious diseases specialist in Japan.

\section{Materials And Methods \\ Study design and patient selection}

This study was conducted at National Hospital Organization Nishisaitama-Chuo National Hospital, a 325bed regional general hospital in Japan, from January 2013 to June 2015. There are no infectious diseases specialists affiliated with the hospital.

The outcomes of the pharmacist-led ASP group, which received pharmacist intervention, and the control group, who did not receive pharmacist intervention and were management to usual treatment, were compared.

The inclusion criterion included patients aged 18 years or older who were diagnosed with gram-negative bacteremia. Meanwhile, the exclusion criteria were immunocompromised patients; those with sepsis, intravascular device, abscesses, carbapenem-resistant Enterobacteriaceae, and carbapenemase-producing Enterobacteriaceae; and those taking antimicrobials for less than 5 days or more than 21 days for suspected non-infectious or complicated bacteremia.

\section{Pharmacist-led ASP}

The pharmacists performed an antimicrobial time-out at 72 hours after blood culture collection and optimized therapy based on the patient's clinical response and test results. Antimicrobial time-out is a technique in which the pharmacist discusses the efficacy, duration, de-escalation, and side effects of antimicrobial therapy on days 3, 5, 7, and 10 with the attending physician. Seven pharmacists (one infectious diseases [ID] pharmacist and six ward pharmacists) were included in the pharmacist-led ASP team.

\section{Outcomes}

The primary outcome was the duration of antibiotic treatment.

The secondary outcomes were de-escalation, clinical success and failure, recurrence, infectious diseases readmission, Clostridioides difficile infection, and 30- and 60-day mortality rates.

\section{Data collection}

We collected data including those of age, sex, estimated creatinine clearance, medical history, Charlson Comorbidity Index [7], acquisition and source of bacteremia, and blood culture isolates. All data were collected from medical charts.

\section{Statistical analysis}

The sample size was calculated by assuming that the pharmacist-led ASP can reduce the mean treatment time (16 days, standard deviation: 2.23 ) for gram-negative bacteremia by 2 days (12.5\%) in the pilot period (January 2013 to June 2013). To account for missing data, the total number of patients in each group was set at 35. Continuous data were analyzed using the Mann-Whitney U test, and categorical data were examined using the Fisher's exact test or the chi-square test. All tests were two-tailed, and a p-value of $<0.05$ was considered statistically significant. All statistical analyses were performed using R version 4.0.3 ( $R$ Foundation for Statistical Computing, Vienna, Austria).

\section{Ethical consideration}

This study was approved by the Research Ethics Committee of National Hospital Organization NishisaitamaChuo National Hospital, Tokorozawa-shi (approval no.: 26-05).

\section{Results}

Figure 1 shows the flowchart of patient selection. In total, 116 patients were assessed. Then 34 and 32 patients were included in the pharmacist-led ASP and control groups, respectively. The background characteristics of the pharmacist-led ASP and control groups were similar (Table 1). 


\section{Cureus}

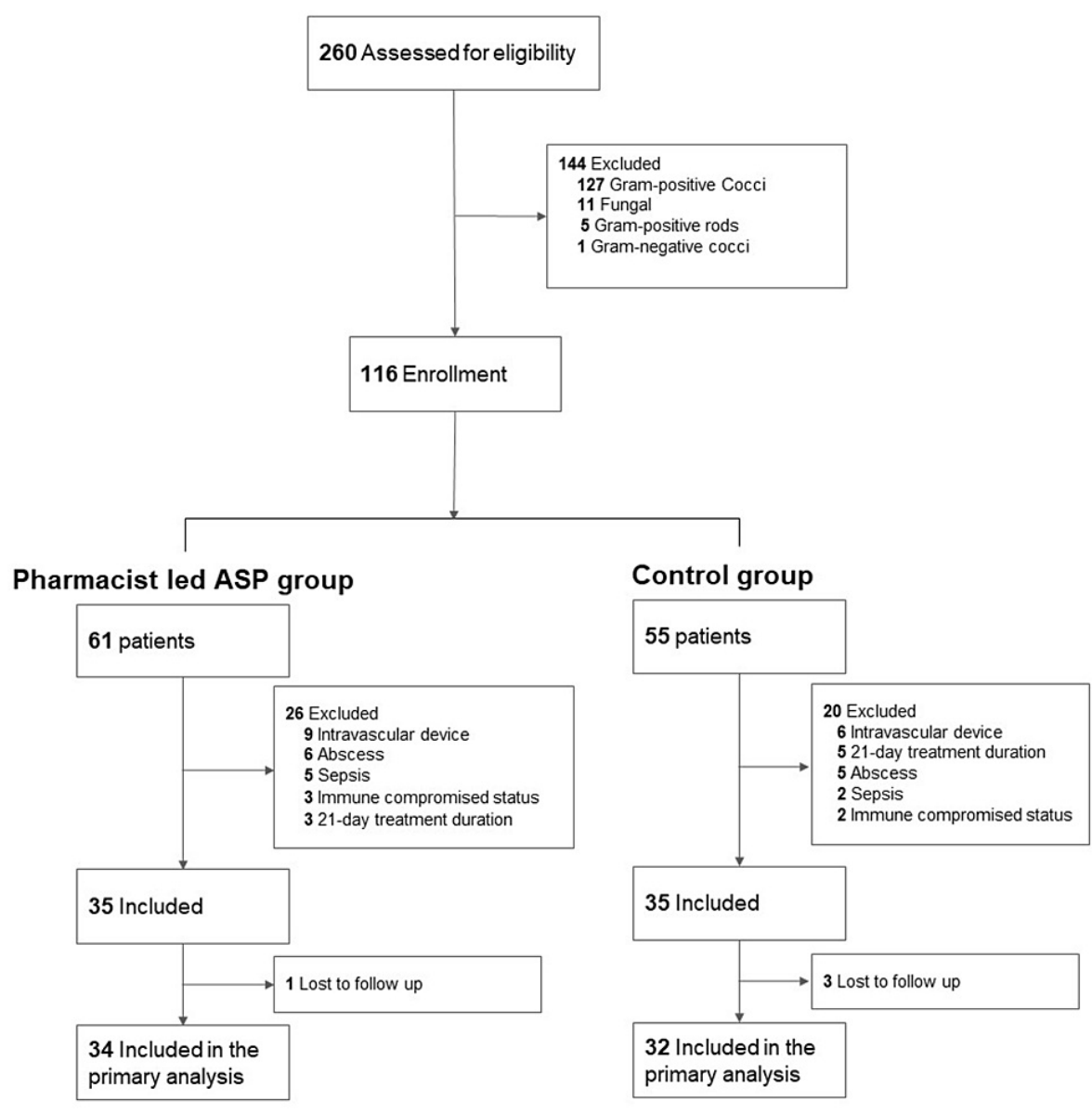

\section{FIGURE 1: Flowchart of patient selection}

In total, 116 patients were assessed. Then, 34 and 32 patients were included in the pharmacist-led ASP and control groups, respectively.

ASP: antimicrobial stewardship program 


\section{Cureus}

\begin{tabular}{|c|c|c|}
\hline & Pharmacist-led ASP group & Control group \\
\hline & $\mathrm{n}=34$ & $\mathrm{n}=32$ \\
\hline Male/female & $20 / 14$ & $18 / 14$ \\
\hline Age (years), median (IQR) & $82(75-84)$ & 79 (74-84) \\
\hline Body weight (kg), median (IQR) & $54(49-66)$ & $56(52-63)$ \\
\hline Estimated creatinine clearance (mL/min), median (IQR) & $47(38-60)$ & $58(36-72)$ \\
\hline \multicolumn{3}{|l|}{ Primary diagnosis } \\
\hline Gastroenterology & 12 & 12 \\
\hline Surgery & 7 & 6 \\
\hline Urology & 7 & 7 \\
\hline Cardiology & 4 & 4 \\
\hline Obstetrics and Gynecology & 2 & 0 \\
\hline Others & 2 & 3 \\
\hline \multicolumn{3}{|l|}{ Comorbidities } \\
\hline Charlson Comorbidity Index, median (IQR) & $1(0-2)$ & $1(0-2)$ \\
\hline Cancer & 6 & 5 \\
\hline Diabetes mellitus & 8 & 8 \\
\hline Heart failure & 2 & 3 \\
\hline Liver cirrhosis & 2 & 2 \\
\hline \multicolumn{3}{|l|}{ Bacteremia acquisition } \\
\hline Community acquired & 18 & 20 \\
\hline Nosocomial & 15 & 12 \\
\hline Unknown & 1 & 0 \\
\hline \multicolumn{3}{|l|}{ Source of bacteremia } \\
\hline Urinary & 26 & 22 \\
\hline Abdominal & 4 & 5 \\
\hline Unknown & 4 & 3 \\
\hline
\end{tabular}

\section{TABLE 1: Baseline characteristics of the participants}

The background characteristics of the pharmacist-led ASP and control groups were similar.

ASP: antimicrobial stewardship program; IQR: interquartile range

Table 2 shows the causative microorganisms. Escherichia coli was the most common causative agent in both groups, and the number of extended-spectrum beta-lactamase (ESBL) cases was similar. 


\section{Cureus}

\begin{tabular}{|c|c|c|}
\hline & Pharmacist-led ASP group & Control group \\
\hline & $\mathrm{n}=34$ & $\mathrm{n}=32$ \\
\hline \multicolumn{3}{|l|}{ Blood culture isolate, no. (\%) } \\
\hline Escherichia coli & $18(52.9)$ & $14(43.8)$ \\
\hline Extended-spectrum beta-lactamase & $2(5.9)$ & $3(9.3)$ \\
\hline Klebsiella spp & $2(5.9)$ & $1(3.1)$ \\
\hline Klebsiella pneumonia & $1(2.9)$ & $3(9.3)$ \\
\hline Extended-spectrum beta-lactamase & $0(0)$ & $1(3.1)$ \\
\hline Klebsiella oxytoca & $3(8.8)$ & $2(6.3)$ \\
\hline Extended-spectrum beta-lactamase & $1(2.9)$ & $0(0)$ \\
\hline Enterobacter spp & $1(2.9)$ & $2(6.3)$ \\
\hline Enterobacter cloacae & $2(5.9)$ & $0(0)$ \\
\hline Proteus spp & $0(0)$ & $1(3.1)$ \\
\hline Acinetobacter baumannii & $1(2.9)$ & $2(6.3)$ \\
\hline Acinetobacter /woffii & $0(0)$ & $1(3.1)$ \\
\hline Others & $3(8.8)$ & $2(6.3)$ \\
\hline
\end{tabular}

\section{TABLE 2: Data about the pathogenic microorganisms detected in multiple blood cultures}

Escherichia coli was the most common causative agent in both groups.

ASP: antimicrobial stewardship program

Figure 2 shows the distribution of the number of days of antimicrobial therapy, and Table 3 depicts the median number of days of antimicrobial therapy and the secondary outcomes of each group. The median number of days of antimicrobial administration was 8 (interquartile range [IQR]: 7-14) days in the pharmacist-led ASP group and 14 (IQR: 10-15) days in the control group. The median number of sources of bacteremia significantly reduced in the pharmacist-led ASP group $(\mathrm{p}<0.001)$. The de-escalation rates were 11 (32.4\%) cases in the pharmacist-led ASP group and 4 (12.5\%) cases in the control group. The pharmacistled ASP group had higher rates than the control group $(p=0.08)$. However, the results did not significantly differ. 


\section{Cureus}

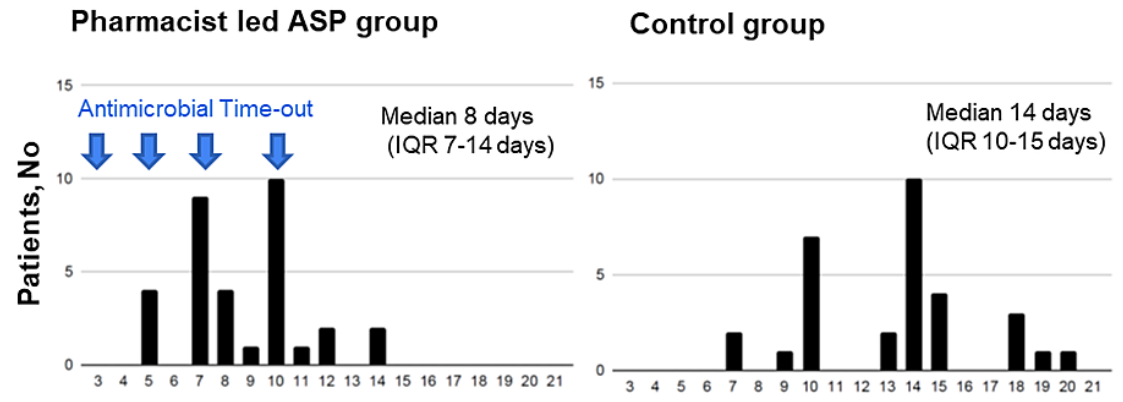

Antibiotic duration, day

FIGURE 2: Distribution of the number of days of antimicrobial therapy

The median number of days of antimicrobial administration was 8 (IQR: 7-14) days in the pharmacist-led ASP group and 14 (IQR: 10-15) days in the control group.

ASP: antimicrobial stewardship program; IQR: interquartile range

\begin{tabular}{|c|c|c|c|}
\hline & Pharmacist-led ASP group & Control group & P-value \\
\hline & $\mathrm{n}=34$ & $\mathrm{n}=32$ & \\
\hline \multicolumn{4}{|l|}{ Primal outcome } \\
\hline Antıbıotıc treatment duratıon (days), median (IQR) & $8(1-10)$ & $14(10-15)$ & $<0.01$ \\
\hline \multicolumn{4}{|l|}{ Secondary outcomes, no. (\%) } \\
\hline De-escalation & $11(32.4)$ & 4 (12.5) & 0.08 \\
\hline Clinical success & $32(94.1)$ & $30(93.8)$ & 1.0 \\
\hline Clinical failure & $2(5.9)$ & $2(6.3)$ & 1.0 \\
\hline Recurrent bacteremia & $0(0)$ & $0(0)$ & 1.0 \\
\hline Infectious diseases re-admission & $0(0)$ & $0(0)$ & 1.0 \\
\hline Clostridioides difficile infection & $1(2.9)$ & $0(0)$ & 1.0 \\
\hline 30-Day mortality & $1(2.9)$ & $1(3.1)$ & 1.0 \\
\hline 60-Day mortality & $2(5.9)$ & $2(6.3)$ & 1.0 \\
\hline
\end{tabular}

\section{TABLE 3: Clinical outcomes}

Depicts the median number of days of antimicrobial therapy and the secondary outcomes of each group.

ASP: antimicrobial stewardship program; IQR: interquartile range

\section{Discussion}

This study had two important findings. First, the number of days of antimicrobial therapy for uncomplicated gram-negative bacteremia decreased in the pharmacist-led ASP group. Second, in a limited sample size, the group did not experience an increase in treatment failure, mortality, relapse, and re-admissions. 
The pharmacist-led ASP was associated with a reduced number of days of antimicrobial therapy for uncomplicated gram-negative bacteremia. We assumed that it can reduce the number of days of antimicrobial therapy by $2(12.5 \%)$ in the pharmacist-led ASP group. However, our results showed that the number of days decreased by 6 . This may be due to the fact that the antimicrobial time-out by the pharmacists prompted the attending physician to reconfirm the general condition of the patient, leading to the decision to discontinue the antimicrobial therapy for patients in good condition.

Previously, von Dach et al. reported that in adult patients with uncomplicated gram-negative bacteremia, both individualized antimicrobial dosing based on C-reactive protein levels and 7-day fixed dosing were not inferior to 14-day fixed dosing in terms of 30-day clinical failure rate [8]. Although this was a randomized controlled trial including 504 patients from multiple centers, the 30-day clinical failure rate was low. However, its usefulness should be interpreted with caution. Therefore, antimicrobial time-out, which is performed by pharmacists, can reduce the risk of adverse drug reactions and can be used in clinical settings.

Further, in a limited sample size, the pharmacist-led ASP was not correlated with increased treatment failure, mortality, relapse, and re-admissions. Brotherton et al. reported that the pharmacist-led $S$. aureus bacteremia management bundles was associated with a lower mortality or 90-day readmission rate [9]. However, our study showed that the pharmacist-led ASP was not associated with a significant reduction in mortality rate because gram-negative rod (GNR) bacteria have a shorter duration of standard antimicrobial therapy compared with gram-positive cocci (GPC) bacteremia and a lower risk of abscess formation including endocarditis. Notably, the reduced duration of antimicrobial therapy may not have a negative effect on the prognosis or adverse effects. Moreover, an increasing trend in de-escalation rates was observed. Previous studies have reported that antimicrobial time-out is correlated with a reduced antimicrobial use and medical cost [10-12], and the combination of these interventions is more effective in reducing cases of resistance and beneficial in terms of costs.

The current study had several limitations. First, patients who were critically ill or had highly resistant strains were excluded. These patients required an individualized approach and thus the data was difficult to generalize. Second, this was a single-center study. We believed it was not statistically appropriate to perform a multivariate analysis based on the number of patients in this study. Third, the different primary diagnoses can be confounding factors. We may need to consider stratification as a response. However, a multicenter study should be conducted, and more cases must be included. In this manner, patient prognosis and safety can be better defined.

\section{Conclusions}

The pharmacist-led ASP was associated with a reduced number of days of antimicrobial therapy for uncomplicated gram-negative bacteremia among patients admitted in a community hospital without an infectious diseases specialist. Hence, pharmacists must discuss not only the type of antimicrobial agents but also the duration of treatment with the attending physician.

However, interpretation is limited by the following factors: lack of inclusion of highly resistant cases, singlecenter study, low observed event rate, such as treatment failure, mortality, relapse, and re-admissions, and the variability in departments.

\section{Additional Information \\ Disclosures}

Human subjects: Consent was obtained or waived by all participants in this study. National Hospital Organization Nishisaitama Chuo Hospital issued approval Approval no.: 26-05. Animal subjects: All authors have confirmed that this study did not involve animal subjects or tissue. Conflicts of interest: In compliance with the ICMJE uniform disclosure form, all authors declare the following: Payment/services info: All authors have declared that no financial support was received from any organization for the submitted work. Financial relationships: All authors have declared that they have no financial relationships at present or within the previous three years with any organizations that might have an interest in the submitted work. Other relationships: All authors have declared that there are no other relationships or activities that could appear to have influenced the submitted work.

\section{References}

1. Schuts EC, Hulscher MEJL, Mouton JW, et al.: Current evidence on hospital antimicrobial stewardship objectives: a systematic review and meta-analysis. Lancet Infect Dis. 2016, 16:847-56. 10.1016/S14733099(16)00065-7

2. Traynor K: CDC says pharmacist-leaders crucial for antimicrobial stewardship . Am J Health Syst Pharm. 2014, 71:689-90. 10.2146/news140033

3. Iwata K: Quantitative and qualitative problems of infectious diseases fellowship in Japan . Int J Infect Dis. 2013, 17:e1098-9. 10.1016/j.ijid.2013.07.009

4. Wenzler E, Wang F, Goff DA, Prier B, Mellett J, Mangino JE, Bauer KA: An automated, pharmacist-driven initiative improves quality of care for Staphylococcus aureus bacteremia. Clin Infect Dis. 2017, 65:194-200. 


\section{Cureus}

10.1093/cid/cix315

5. Arensman K, Dela-Pena J, Miller JL, et al.: Impact of mandatory infectious diseases consultation and realtime antimicrobial stewardship pharmacist intervention on Staphylococcus aureus bacteremia bundle adherence. Open Forum Infect Dis. 2020, 7:ofaa184. 10.1093/ofid/ofaa184

6. Fukuda T, Watanabe H, Ido S, Shiragami M: Contribution of antimicrobial stewardship programs to reduction of antimicrobial therapy costs in community hospital with 429 Beds --before-after comparative two-year trial in Japan. J Pharm Policy Pract. 2014, 7:10. 10.1186/2052-3211-7-10

7. Charlson ME, Pompei P, Ales KL, MacKenzie CR: A new method of classifying prognostic comorbidity in longitudinal studies: development and validation. J Chronic Dis. 1987, 40:373-83. 10.1016/00219681(87)90171-8

8. von Dach E, Albrich WC, Brunel AS, et al.: Effect of C-reactive protein-guided antibiotic treatment duration, 7-day treatment, or 14-day treatment on 30-day clinical failure rate in patients with uncomplicated gramnegative bacteremia: a randomized clinical trial. JAMA. 2020, 323:2160-9. 10.1001/jama.2020.6348

9. Brotherton AL, Rab S, Kandiah S, Kriengkauykiat J, Wong JR: The impact of an automated antibiotic stewardship intervention for the management of Staphylococcus aureus bacteraemia utilizing the electronic health record. J Antimicrob Chemother. 2020, 75:1054-60. 10.1093/jac/dkz518

10. Wolfe JR, Bryant AM, Khoury JA: Impact of an automated antibiotic time-out alert on the de-escalation of broad-spectrum antibiotics at a large community teaching hospital. Infect Control Hosp Epidemiol. 2019, 40:1287-9. 10.1017/ice.2019.197

11. Wirtz AL, Burns AN, Lee BR, et al.: Effectiveness and safety of mandatory antimicrobial indications and durations and a pharmacist-driven 48-hour time-out in a pediatric hospital. Am J Health Syst Pharm. 2020, 77:614-21. 10.1093/ajhp/zxaa029

12. Lee TC, Frenette C, Jayaraman D, Green L, Pilote L: Antibiotic self-stewardship: trainee-led structured antibiotic time-outs to improve antimicrobial use. Ann Intern Med. 2014, 161:S53-8. 10.7326/M13-3016 